\title{
Involvement of two positively charged residues of Chlamydomonas reinhardtii glyceraldehyde-3-phosphate dehydrogenase in the assembly process of a bi-enzyme complex involved in $\mathrm{CO}_{2}$ assimilation
}

\author{
Emmanuelle Graciet ${ }^{1 *}$, Guillermo Mulliert ${ }^{\mathbf{2}}$, Sandrine Lebreton ${ }^{\mathbf{1}}$ and Brigitte Gontero ${ }^{1}$ \\ ${ }^{1}$ Laboratoire Génétique et Membranes, Département Biologie Cellulaire, Institut Jacques Monod, UMR 7592 CNRS, Universités \\ Paris VI-VII, Paris; ${ }^{2}$ Laboratoire de cristallographie et de modélisation des matériaux minéraux et biologiques (UMR 7036), \\ Faculté des Sciences et Techniques, Vandoeuvre-lès-Nancy, France
}

\begin{abstract}
The glyceraldehyde-3-phosphate dehydrogenase (GAPDH) in the chloroplast of Chlamydomonas reinhardtii is part of a complex that also includes phosphoribulokinase (PRK) and CP12. We identified two residues of GAPDH involved in protein-protein interactions in this complex, by changing residues K128 and R197 into A or E. K128A/E mutants had a $K_{\mathrm{m}}$ for NADH that was twice that of the wild type and a lower catalytic constant, whatever the cofactor. The kinetics of the mutant R197A were similar to those of the wild type, while the R197E mutant had a lower catalytic constant with NADPH. Only small structural changes near the mutation may have caused these differences, since circular dichroism and fluorescence spectra were similar to those of wild-type GAPDH. Molecular modelling of the mutants led to the same conclusion. All mutants, except R197E, reconstituted the GAPDH-CP12 subcomplex. Although the dissociation
\end{abstract}

constants measured by surface plasmon resonance were 10-70-fold higher with the mutants than with wild-type GAPDH and CP12, they remained low. For the R197E mutation, we calculated a $4 \mathrm{kcal} / \mathrm{mol}$ destabilizing effect, which may correspond to the loss of the stabilizing effect of a salt bridge for the interaction between GAPDH and CP12. All the mutant GAPDH-CP12 subcomplexes failed to interact with PRK and to form the native complex. The absence of kinetic changes of all the mutant GAPDH-CP12 subcomplexes, compared to wild-type GAPDH-CP12, suggests that mutants do not undergo the conformation change essential for PRK binding.

Keywords: phosphoribulokinase; glyceraldehyde-3-phosphate dehydrogenase; CP12; site-directed mutagenesis; protein-protein interactions.
Several lines of evidence point to the involvement of supramolecular complexes in the Benson-Calvin cycle, responsible for $\mathrm{CO}_{2}$ assimilation in photosynthetic organisms [1-5]. Even though interactions between proteins are involved in nearly all biological functions, the physicochemical principles governing the interaction of proteins are not fully understood.

In the literature, two types of complexes are defined [6,7]: obligatory or permanent ones, whose constituents only exist as part of complexes, and transitory complexes, whose components are found either under an associated or an individual state. Transitory interactions are dynamic processes characterized by equilibrium constants and therefore

Correspondence to B. Gontero, Laboratoire Génétique et Membranes, Département Biologie Cellulaire, Institut Jacques Monod, UMR 7592 CNRS, Universités Paris VI-VII, 2 place Jussieu, 75251 Paris cedex 05, France. Fax: + 331 44275994, Tel.: + 33144274719 ,

E-mail: meunier@ijm.jussieu.fr

Abbreviations: BPGA, 1,3-biphosphoglyceric acid; GADPH, glyceraldehyde-3-phosphate dehydrogenase; PDB, Protein Data Bank; PRK, phosphoribulokinase.

* Present address: California Institute of Technology, Division of Biology, 147-75, 1200 East California Blvd., Pasadena CA 91125, USA.

(Received 19 September 2004, revised 7 October 2004, accepted 13 October 2004) depend on the in vivo relative concentration of the different components. This dynamics may explain why a given protein is described in the literature as part of protein complexes having different compositions. Different isolation procedures could also explain the discrepancies in the published compositions of some protein complexes [8,9]. The physico-chemical properties of the interface of obligatory and transitory complexes have been characterized by studying the structure of complexes deposited in the Protein Data Bank (PDB) [10]. The interface of obligatory complexes is rich in hydrophobic residues and greatly resembles the buried parts of the protein $[11,12]$. On the contrary, the interface of transitory complexes bears many charged residues, and its composition is closer to that of solvent-exposed regions of the protein. The arginine residue seems to be more frequent at the interface of proteins in transitory complexes [13].

We have isolated from the green alga Chlamydomonas reinhardtii a bi-enzyme complex $(460 \mathrm{kDa})$ which is made up of two molecules of tetrameric glyceraldehyde-3-phosphate dehydrogenase (GAPDH) (EC 1.2.1.13), two molecules of dimeric phosphoribulokinase (PRK) (EC 2.7.1.19) and of a small flexible protein involved in the assembly of this complex, CP12 [5,14,15]. When this GAPDH-CP12PRK complex is dissociated by dilution or strong reducing conditions, GAPDH is released as a tetrameric $\mathrm{A}_{4}$ form associated with CP12 (native GAPDH), while PRK is released under an isolated homodimeric form. We have 
previously shown that protein-protein interactions can result in information transfer, imprinting effects and can modify the regulatory properties of the enzymes involved in this complex [16-20].

GAPDH and PRK are known to be involved in transitory interactions $[1,3,14,21-23]$, but the residues essential for these interactions remain unknown. In the past [24], we have shown that the conserved residue arginine 64 of $C$. reinhardtii PRK is involved in the interaction of this enzyme with the GAPDH-CP12 subcomplex. This report describes the behaviour of four GAPDH mutants to explore the specific interactions between GAPDH and CP12, and then between this subcomplex and PRK.

Lastly, few data are available for the thermodynamics of the association reactions in higher order structures. They are based on mutagenesis and binding studies of relatively few complexes $[25,26]$. As the affinities of the mutant GAPDHs for $\mathrm{CP} 12$ can be accurately measured under equilibrium binding conditions using surface plasmon resonance [27], we used this method to assess the apparent contribution of the mutated residues to the formation of the complex.

\section{Experimental procedures}

\section{Materials}

Most chemicals [ATP, NAD $(\mathrm{P}) \mathrm{H}]$ and enzymes (phosphoglycerate kinase) were supplied by Sigma. Blue Sepharose ${ }^{\mathrm{TM}}$ 6 Fast flow was from Amersham Pharmacia Biotech AB, Uppsala, Sweden.

\section{Enzyme purification}

Recombinant wild-type GAPDH and CP12 were purified to apparent homogeneity, as previously described [28,29]. Mutant GAPDHs were purified using a Blue Sepharose ${ }^{\mathrm{TM}}$ 6 Fast flow step [28] with $30 \mathrm{~mm}$ Tris, 4 mм EDTA, $0.1 \mathrm{~mm}$ NAD, $2.5 \mathrm{~mm}$ dithiothreitol, $\mathrm{pH} 7.5$ as equilibration buffer (buffer A). Purified active mutant GAPDHs were eluted with buffer A supplemented with $0.5 \mathrm{M} \mathrm{NaCl}$. All purified GAPDHs were stored at $-80{ }^{\circ} \mathrm{C}$ in $10 \%$ aqueous glycerol.

\section{Site-directed mutagenesis}

In vitro mutagenesis was performed using QuickChange ${ }^{\mathrm{TM}}$ site-directed mutagenesis kit (Stratagene). All the mutations were confirmed by sequencing.

\section{Enzyme assays and protein measurements}

The NADH- or NADPH-dependent activities of GAPDH were determined [30] using 1,3-biphosphoglyceric acid (BPGA) formed in a mixture containing $35 \mathrm{~mm}$ ATP, $70 \mathrm{~mm}$ phosphoglyceric acid and $30 \mathrm{U}$ phosphoglycerate kinase, incubated at $30{ }^{\circ} \mathrm{C}$ for $30 \mathrm{~min}$. The BPGA concentration was spectrophotometrically determined and found to be $15 \pm 3 \mathrm{~mm}$. Activities were recorded using a UV2 Pye Unicam spectrophotometer. Experimental data were fitted to theoretical curves using SIGMA PLOT 5.0, V5. GAPDH activities measured at constant cofactor $[\mathrm{NAD}(\mathrm{P}) \mathrm{H}]$ concentration and varied concentrations of the substrate (BPGA) were fitted to a sigmoid curve:

$$
\frac{\mathrm{v}}{[\mathrm{E}]_{0}}=\mathrm{k}_{\mathrm{cat}} \times\left(\frac{[\mathrm{BPGA}]^{\mathrm{n}_{\mathrm{h}}}}{\mathrm{K}_{0.5}^{\mathrm{n}_{\mathrm{h}}}+[\mathrm{BPGA}]^{\mathrm{n}_{\mathrm{h}}}}\right)
$$

where, $k_{\text {cat }}$ is the catalytic constant, $\mathrm{n}_{\mathrm{h}}$ the Hill coefficient and $K_{0.5}$ the BPGA concentration for which half the maximal velocity is obtained. GAPDH activities measured at constant BPGA concentration and varied concentrations of $\mathrm{NAD}(\mathrm{P}) \mathrm{H}$ were fitted to a hyperbola according to Michaelis-Menten kinetics.

Protein concentration was assayed with the Bio-Rad protein dye assay reagent, using bovine serum albumin as a standard [31].

\section{Molecular modelling}

Modeller 6v2 [32] was used to make a model of the tetrameric GAPDH from C. reinhardtii based on the structure of the GAPDH from Bacillus stearothermophilus (PDB code 1 GD1). The resulting structure was minimized and a molecular dynamics was made with AMBER 6.0 [33]. The four mutants (K128A, K128E, R197A and R197E) were constructed in silico from the average structure of molecular dynamics and were minimized with AMBER 6.0. To model the position of NADH and of NADPH, these substrates were initially docked in the same position as the NAD of 1 GD1. Parameters for both cofactors were taken from the AMBER web site. The 10 structures were minimized in a $20 \AA$ radius from the substrate in only one monomer.

\section{Aggregation states of the enzymes}

The formation of the GAPDH-CP12 or GAPDH-CP12PRK complex was checked by native PAGE performed on 4-15\% minigels using a Pharmacia Phastsystem apparatus. Proteins were transferred to nitrocellulose filters $(0.45 \mu \mathrm{m}$, Schleicher and Schüll) by passive diffusion for $16 \mathrm{~h}$. The filters were then immunoblotted with a rabbit antiserum directed against recombinant $C$. reinhardtii CP12 (1 : 2000) or a rabbit antiserum directed against recombinant C. reinhardtii GAPDH (1:5000). Antibody binding was revealed using alkaline phosphatase [34]. For GAPDHCP12-PRK reconstitution assays [29], a rabbit antiserum directed against recombinant spinach PRK (1:1000) was used.

\section{Biosensor assays}

Purified CP12 (50 $\left.\mu \mathrm{g} \cdot \mathrm{mL}^{-1}\right)$ was coupled to carboxymethyl dextran (CMD)-coated biosensor chip (CM5, BiaCore) following the manufacturer's instructions. We studied the interaction of wild-type or mutant recombinant GAPDHs to immobilized oxidized CP12 using HBS running buffer (BiaCore) supplemented with $0.1 \mathrm{~mm}$ NAD, $5 \mathrm{~mm}$ Cys, $\mathrm{pH} 7.5$ at $20 \mu \mathrm{L} \cdot \mathrm{min}^{-1}$. Different concentrations of GAPDH were injected (analyte). The analyte interacts with the ligand (CP12) to give the association phase, then the analyte begins to dissociate as soon as injection is stopped and replaced by buffer. The observed curves were fitted assuming single phase kinetics (single phase dissociation/ association). The kinetic parameters were calculated from these fits using the BiaEvaluation software (v2.1, BiaCore). 


\section{Results}

\section{Rationale for the mutation of residues Lys128 and Arg197}

Like all GAPDHs (chloroplast and glycolytic), the $\mathrm{A}_{4}$ chloroplast GAPDH is made up of two functional domains, one corresponding to the cofactor-binding domain, or
Rossman fold (residues 1-147 and 313-334 in spinach GAPDH (accession code in PDB:1JN0), the other being the catalytic domain (residues 148-313). The latter comprises the S loop (residues 177-203) that is close to the NADP nicotinamide moiety [35].

The structure of wild-type C. reinhardtii GAPDH obtained by molecular modelling (Fig. 1A), and that of chloroplast spinach GAPDH [35] were examined to

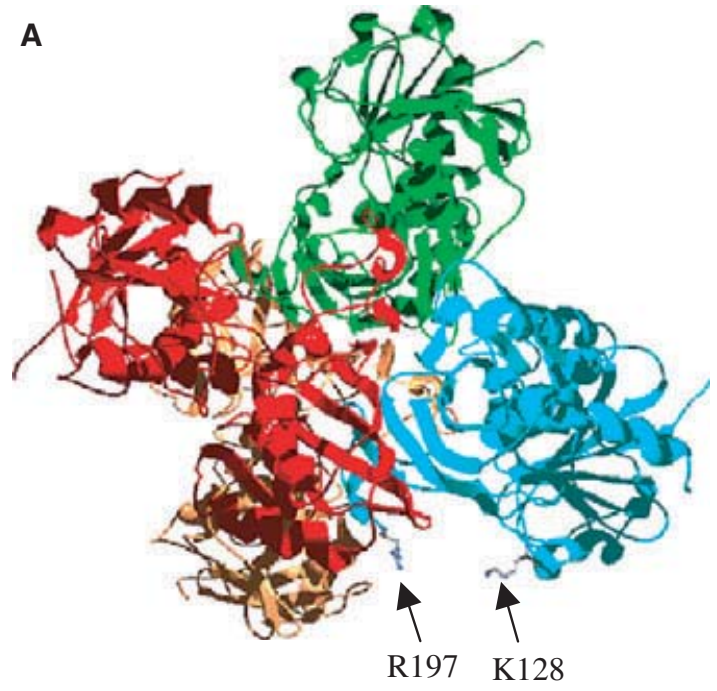

B

AthalianaB 119 V I I T A P A K G A D I P T Y VMG VNEQDYGHDVAN I I S NASCT T N

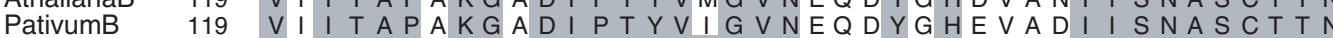

SoleraceaB 119 V I I T A P A K G S I P T Y V VG VNEKDYGHDVAN I I S NASCTTN

NtabacumB 119 VI I APAKGAD I PTYVVGVNEQDYSHEVADI I SNASCTTN

A.thalianaA 119 VI I T APGKG - D I P T Y VVGVNADAYSHDEP-I I S NASCTTN

PsativumA 120 VLI TAPGKG-DI PTYVVGVNADAYTHADD - I I S NASCTT N

SoleraceaA 119 VLI T APGKG - D I PTY T VGVNEEGYTHADT - I I S NASCTTN

Chlamy 121 VL I T A P A KDKD I P TFVVGVNEGDYKHEYP - I I SNASCT T N

Synechocystis 118 V L I T A P G K G P I G T Y V VGVNAHEYKHEEYEVI SNASCTT N

Synechococcust 19 VLI T A PGKGEGVGTYVIGVNDSEYRHEDFAVI S NASCT T N

AthalianaB 159 CLAPF A K VLDEEFG I VKG TMTTTHSYTGDQRLLDASHRDL

PativumB $\quad 159$ CLAPFAKVLDEEFG I VKGTMTTTHSYTGDQRLLDASHRDL

SoleraceaB 159 CLAPF VKVLDEELG IVKGTMTTTHSYTGDQRLLDASHRDL

NtabacumB 159 CLAPF V K VMDEELG IVKGTMTTTHSYTGDQRLLDASHRDL

A.thalianaA 157 CLAPFVKVLDQKFG I I KGTMTTTHSYTGDQRLLDASHRDL

PsativumA 158 CLAPF VKVLDQKFG I I KGTMTTTHSYTGDQRLLDASHRDL

SoleraceaA 157 CLAPFVKVLDQKFG I I KGTMTTTHSYTGDQRLLDASHRDL

Chlamy $\quad 160$ CLAPFVKVLEQKFG I VKGTMTT THSYTGDQRLLDASHRDL

Synechocystis 158 CLAPFGKVINDNFG I I KGTMTTTHSYTGDQR I LDASHRDL

Synechococcusi59 CLAPVAKVLHDNFGI I KGTMTTTHSYTLDQR I LDASHRDL

AthalianaB 199 RRARA A L N I V P T S T G A A K A V SL VLPQLKGKL NG I A L R P P

PativumB 199 RRARA A A N I VPTSTGA A K A VSLVLPQLKGKLNGI ALRVP

SoleraceaB 199 RRARA A A L N I V P T S T G A A K A V SL VLPQLKGKLNG I ALR R P

NtabacumB 199 RRARA A A N I VPT STGA A K A V SL VLPQLKGKLNG I ALRVP

A.thalianaA 197 RRARAAALNIVPTSTGAAKAVALVLPNLKGKLNGIALRVP

PsativumA 198 RRARAAALNIVPTSTGAAKAVALVLPTLKGKLNGIALRVP

SoleraceaA 197 RRARA A CLN I VPTSTGA A KA VALVLPNLKGKLNGIALRVP

Chlamy 200 RRARAAALN IVPTT TGA A KAVSLVLPSLKGKLNGIALRVP

Synechocystis 198 RRARA A A VN I VPTSTGA A K A V ALVI PELQGKLNGIALRVP

Synechococcusis9 RRARAAAVNIVPTTTGAAKAVALVI PELKGKLNGIALRVP

Fig. 1. Modelled structure of $C$. reinhardtii GAPDH and amino acid comparison with other GAPDHs. (A) Localization and orientation of residues K128 and R197 of C. reinhardtii GAPDH. Ribbon model of the photosynthetic $\mathrm{A}_{4}$ GAPDH tetramer in which residues corresponding to K128 and $\mathrm{R} 197$ in C. reinhardtii GAPDH are situated in a groove between two monomers. The $\mathrm{O}$ monomer is represented in cyan, the $\mathrm{P}$ in red, the $\mathrm{Q}$ in green and the R monomer in orange. (B) Partial amino acid sequence alignment of chloroplast GAPDHs. Alignment was performed with CLUSTALw. The residues K128 and R197 (C. reinhardtii numbering) are indicated by arrows. The S loop is underlined. 
determine which residues were accessible to the solvent and could thus be potentially involved in the interaction with the other partners of the GAPDH-CP12-PRK complex. The model of wild-type GAPDH from $C$. reinhardtii, like the structure of spinach GAPDH, shows the presence of a groove containing two positively charged residues, Lys 128 and Arg197 (C. reinhardtii numbering, corresponding to Lys122 and Arg191 in spinach) that seem to protrude and could hence play a role in protein-protein interactions (Fig. 1A). Hydrophobicity distribution patterns were also analyzed using a simple method to identify residues potentially involved in protein-protein interactions [13] This method indicates that among other candidates, residues Lys128 and Arg197 may be involved in proteinprotein interactions. These residues being also conserved among other chloroplast GAPDHs (Fig. 1B), we mutated them in either alanine or glutamic acid.

\section{Kinetic parameters of the R197A and R197E mutant GAPDHs}

The R197A mutant was not significantly different from the wild-type recombinant enzyme. Like the wild-type enzyme, the R197E mutant followed Michaelis-Menten kinetics with NADH and NADPH, but the catalytic rate constant using NADPH was only half that of the wild type. The catalytic efficiency, expressed as $k_{\mathrm{cat}} / K_{\mathrm{m}}$, of the R197E mutant using NADPH was then about one-half $\left(6.3 \mathrm{~s}^{-1} \cdot \mu \mathrm{mol}^{-1}\right)$ that of the wild-type recombinant GAPDH $\left(15.3 \mathrm{~s}^{-1} \cdot \mu \mathrm{mol}^{-1}\right)$. The catalytic efficiency using NADH was not affected. Like the wild type, the mutant showed allosteric behaviour toward BPGA and its $K_{0.5}$ was twofold higher, whatever the cofactor. The kinetic parameters of the purified R197 mutant enzymes and those of the recombinant wild-type enzyme are shown in Tables 1 and 2.

Circular dichroism and fluorescence spectra of the R197A and R197E mutants indicate that the mutations do not change significantly the global structure of the enzyme, compared to the wild-type GAPDH. Molecular modelling also suggests that the interactions between the enzyme and the NADH or NADPH moiety were conserved with both R197 mutants, indicating that both cofactors, either NADH or NADPH, have a correct position in the active site. The overall conformation of each mutant monomer remains essentially similar to that of wild-type GAPDH; root square mean distance values for the superimposition of the $\mathrm{C}^{\alpha}$ atoms of the latter with those

Table 1. Kinetic parameters of mutant GAPDH R197A and R197E, compared to those of the wild-type recombinant enzyme. BPGA concentration was kept constant at $1 \mathrm{~mm}$ and $\mathrm{NAD}(\mathrm{P}) \mathrm{H}$ concentration varied from 0 to $0.25 \mathrm{~mm}$. The concentration of enzyme in the cuvette was $3 \mathrm{~nm}$ with NADPH and $10 \mathrm{~nm}$ with NADH. Kinetic parameters were obtained by fitting the experimental points to a hyperbola, according to Michaelis-Menten kinetics.

\begin{tabular}{lllll}
\hline & {$[\mathrm{NADPH}]$} & & {$[\mathrm{NADH}]$} & \\
& $K_{\mathrm{m}}(\mu \mathrm{M})$ & $k_{\mathrm{cat}}\left(\mathrm{s}^{-1}\right)$ & \multicolumn{1}{c}{$K_{\mathrm{m}}(\mu \mathrm{M})$} & $k_{\mathrm{cat}}\left(\mathrm{s}^{-1}\right)$ \\
\hline Wild-type GAPDH & $28 \pm 3$ & $430 \pm 17$ & $120 \pm 11$ & $104 \pm 3$ \\
R197E GAPDH & $35 \pm 5$ & $220 \pm 10$ & $110 \pm 10$ & $137 \pm 5$ \\
R197A GAPDH & $28 \pm 5$ & $392 \pm 21$ & $99 \pm 26$ & $108 \pm 13$ \\
\hline
\end{tabular}

Table 2. Kinetic parameters of mutant GAPDH R197A and R197E, compared to those of the wild-type recombinant enzyme. $\mathrm{NAD}(\mathrm{P}) \mathrm{H}$ concentration maintained equal to $0.25 \mathrm{~mm}$, while BPGA concentration in the reaction mixture varied from 0 to $2 \mathrm{~mm}$. The concentration of enzyme in the cuvette was as in Table 1. The experimental points were fitted to the equation of a sigmoid (1).

\begin{tabular}{lllll}
\hline & \multicolumn{5}{c}{$K_{0.5}(\mu \mathrm{M})$} & & \\
& Cofactor & BPGA & $n_{\text {Hill }}$ & $k_{\text {cat }}\left(\mathrm{s}^{-1}\right)$ \\
\hline \multirow{2}{*}{ Wild-type GAPDH } & NADPH & $250 \pm 17$ & $1.5 \pm 0.1$ & $430 \pm 17$ \\
& NADH & $95 \pm 10$ & $1.3 \pm 0.1$ & $88 \pm 4$ \\
R197E GAPDH & NADPH & $438 \pm 8$ & $1.4 \pm 0.2$ & $216 \pm 22$ \\
& NADH & $208 \pm 32$ & $1.4 \pm 0.2$ & $96 \pm 7$ \\
R197A GAPDH & NADPH & $254 \pm 25$ & $1.3 \pm 0.1$ & $367 \pm 20$ \\
& NADH & $109 \pm 7$ & $1.9 \pm 0.2$ & $80 \pm 3$ \\
\hline
\end{tabular}

of R197A and R197E were 0.39 and $0.43 \AA$, respectively (data not shown).

\section{Kinetic parameters of the K128A and K128E mutant GAPDHs}

The two GAPDH mutants behaved in a Michaelis-Menten fashion toward the cofactors as does the wild-type enzyme. The $K_{\mathrm{m}}$ for NADH was significantly higher (at least twofold), even though it was not possible to have an accurate estimation of its value due to limitations of the spectrophotometer (standard errors of 20\%). The catalytic rate constants of these mutants with both cofactors were one-half those of the wild-type recombinant enzyme $\left(\sim 7 \mathrm{~s}^{-1} \cdot \mu \mathrm{mol}^{-1}\right)$ using NADPH and about one quarter $\left(\sim 0.2 \mathrm{~s}^{-1} \cdot \mu \mathrm{mol}^{-1}\right)$ in the presence of NADH. For the reason mentioned above, only the $K_{0.5}$ toward BPGA at constant NADPH concentration was further characterized. Fitting the curves with a multifit function using a common value of $K_{0.5}$ for the wild-type and the mutants, and different values of $k_{\text {cat }}$ showed that the small difference in the $K_{0.5}$ values obtained for the mutant GAPDHs was not significant. Specific values for these parameters are given in Tables 3 and 4 .

Again, circular dichroism and fluorescence experiments indicate that the overall structure of these mutants is not different from that of the wild type. The rsmd values obtained for the superimposition of the $\mathrm{C}^{\alpha}$ atoms of the wild-type GAPDH with those of $\mathrm{K} 128 \mathrm{~A}$ and $\mathrm{K} 128 \mathrm{E}$ also

Table 3. Kinetic parameters obtained for the mutants K128A and K128E. BPGA concentration was kept constant at $1 \mathrm{~mm}$ and $\mathrm{NAD}(\mathrm{P}) \mathrm{H}$ concentration varied from 0 to $0.25 \mathrm{~mm}$. The concentration of enzyme in the cuvette was $5 \mathrm{~nm}$ with NADPH and $14 \mathrm{~nm}$ with NADH. Kinetic parameters were obtained by fitting the experimental points to a hyperbola, according to Michaelis-Menten kinetics.

\begin{tabular}{|c|c|c|c|c|}
\hline & \multicolumn{2}{|c|}{ [NADPH] } & \multicolumn{2}{|l|}{ [NADH] } \\
\hline & $K_{\mathrm{m}}(\mu \mathrm{M})$ & $k_{\text {cat }}\left(\mathrm{s}^{-1}\right)$ & $K_{\mathrm{m}}(\mu \mathrm{M})$ & $k_{\text {cat }}\left(\mathrm{s}^{-1}\right)$ \\
\hline Wild-type GAPDH & $28 \pm 3$ & $430 \pm 17$ & $120 \pm 11$ & $104 \pm 3$ \\
\hline K128E GAPDH & $23 \pm 2$ & $161 \pm 5$ & $272 \pm 32$ & $56 \pm 4$ \\
\hline K128A GAPDH & $27 \pm 1$ & $220 \pm 20$ & $250 \pm 50$ & $40 \pm 4$ \\
\hline
\end{tabular}


Table 4. Kinetic parameters obtained for the mutants $\mathrm{K} 128 \mathrm{~A}$ and K128E. NAD(P)H concentration maintained equal to $0.25 \mathrm{~mm}$, while BPGA concentration in the reaction mixture varied from 0 to $2 \mathrm{~mm}$. The concentration of enzyme in the cuvette was as in Table 3. The experimental points were fitted to the equation of a sigmoid (Eqn 1).

\begin{tabular}{lllll}
\hline & \multicolumn{5}{c}{$K_{0.5}(\mu \mathrm{M})$} & & \\
& Cofactor & BPGA & $n_{\text {Hill }}$ & $k_{\text {cat }}\left(\mathrm{s}^{-1}\right)$ \\
\hline Wild-type GAPDH & NADPH & $250 \pm 17$ & $1.5 \pm 0.1$ & $430 \pm 17$ \\
& NADH & $95 \pm 10$ & $1.3 \pm 0.1$ & $88 \pm 4$ \\
K128E GAPDH & NADPH & $322 \pm 38$ & $1.7 \pm 0.2$ & $159 \pm 11$ \\
& NADH & n. d. & n. d. & n. d. \\
K128A GAPDH & NADPH & $370 \pm 80$ & $1.4 \pm 0.3$ & $225 \pm 25$ \\
& NADH & n. d. & n. d. & n. d. \\
\hline
\end{tabular}

n.d., not done.

suggest no strong differences between the monomers (rsmd values were 0.4 and $0.45 \AA$ A, respectively).

\section{GAPDH-CP12 and GAPDH-CP12-PRK reconstitution experiments}

Reconstitution experiments were performed using equimolar proportions of GAPDH and CP12 to see whether the mutant GAPDHs were able to reconstitute the GAPDHCP12 complex as did the wild-type enzyme. After incubation during $16 \mathrm{~h}$ at $4{ }^{\circ} \mathrm{C}$, the formation of the GAPDHCP12 complex was assessed by native PAGE followed by incubation with the anti-CP12 and anti-GAPDH antibodies (Fig. 2). The GAPDH-CP12 complex was reconstituted in vitro with all mutants except R197E.

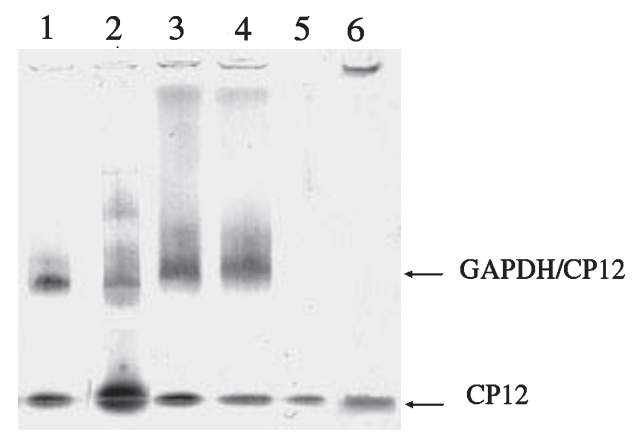

Fig. 2. Western blot analysis of the in vitro reconstitution of the recombinant GAPDH-CP12 complex. Aliquots from the reconstitution mixture were separated on a $4-15 \%$ gradient native gel. The proteins were transferred on a nitrocellulose membrane and probed with anti-C. reinhardtii CP12 (lanes 1, 2, 3, 4- reconstitution mixtures with mutants K128E, K128A, R197A and wild-type recombinant GAPDH, respectively, lane 5-80 ng of CP12 alone). We checked that CP12 antibodies did not cross-react with recombinant GAPDH. For all reconstitution experiments, equimolar proportions of GAPDH and CP12 were used. In lanes 1,3 and $4,1 \mu \mathrm{g}$ of GAPDH $(\sim 0.08 \mathrm{nmol})$ and $0.08 \mu \mathrm{g}$ of CP12 $(\sim 0.08 \mathrm{nmol})$ were mixed and $1 \mu \mathrm{g}$ of the mixture was analyzed. In lane $2,38 \mu \mathrm{g}$ of the K128A mutant and $3 \mu \mathrm{g}$ of $\mathrm{CP} 12$ were mixed and about $10 \mu \mathrm{g}$ of the mixture was analyzed. The same conditions as in lane 2 were used for the reconstitution experiment using the R197E mutant, but the band corresponding to the GAPDH-CP12 subcomplex was absent (lane 6).
Those mutants that formed the GAPDH-CP12 subcomplex were further checked for their ability to reconstitute the GAPDH-CP12-PRK complex under conditions favourable for the wild-type recombinant GAPDH. The GAPDH-CP12-PRK complex was not reconstituted (data not shown), showing that none of the mutants tested acted normally regarding the interaction between the GAPDHCP12 subcomplexes and PRK.

We have previously shown that the $k_{\text {cat }}$ of the GAPDHCP12 complex formed when wild-type GAPDH associates with $\mathrm{CP} 12$, decreased after $45 \mathrm{~min}$ at $30{ }^{\circ} \mathrm{C}$, to become equal to that of the native GAPDH. After $16 \mathrm{~h}$ at $4{ }^{\circ} \mathrm{C}$, the $K_{0.5}$ for the substrate also became equal to that of the native enzyme. These kinetic changes were assumed to be linked to conformation changes upon association of GAPDH with CP12 [28], which would be essential for the binding of PRK and assembly of the complex [29]. The same kinetic experiments were performed with the GAPDH-CP12 complexes obtained with the mutant GAPDHs to see whether the lack of complex reconstitution could be linked to the absence of conformation changes when GAPDH and CP12 associated. The mutant GAPDH-CP12 complexes showed allosteric behaviour with respect to BPGA whatever the cofactor used, as did the wild-type GAPDH-CP12 complex. However, no change, either in the $K_{0.5}$-values or in the catalytic rate constants, was observed (data not shown).

\section{Biacore experiments}

The interactions between mutant GAPDHs and CP12 were further characterized by surface plasmon resonance (BiaCore). The sensorgrams are reported in Fig. 3. The calculated dissociation constants $\left(K_{\mathrm{d}}\right)$ values are summarized in Table 5.

The $K_{\mathrm{d}}$ for R197A, K128E and K128A mutant GAPDHs was found to be in the range of 6-38 nM, compared with $0.44 \mathrm{~nm}$ for the wild-type recombinant GAPDH. The $K_{\mathrm{d}}$ for the R197E mutant dramatically increased $(\sim 275 \mathrm{nM})$. These values allow us to calculate the free energy of the binding of GAPDH to CP12:

$$
\Delta G_{\mathrm{b}}=-\mathrm{RT} \ln K_{\mathrm{d}}
$$

The dissociation constants of mutants and wild-type GAPDHs with CP12 allow the calculation of the difference $\Delta \Delta G_{\mathrm{b}}$ (Eqn 3) and thus quantify the destabilization of the interaction between GAPDH and CP12 that could be directly linked to the point mutations introduced in GAPDH (Table 5).

$$
\Delta \Delta G_{\mathrm{b}}=\Delta G_{\mathrm{b}}^{\mathrm{WT}}-\Delta G_{\mathrm{b}}^{\mathrm{mut}}=-\mathrm{RT} \ln \frac{K_{\mathrm{d}}^{\mathrm{WT}}}{K_{\mathrm{d}}^{\mathrm{mut}}}
$$

The higher effect was observed with the R197E mutant that was previously shown to be incapable of forming the GAPDH-CP12 subcomplex.

\section{Discussion}

Analysis of the structure of $C$. reinhardtii chloroplast GAPDH obtained by molecular modelling and that of spinach $\mathrm{A}_{4}$ GAPDH has led us to mutate the conserved residues Lys128 and Arg197 of C. reinhardtii chloroplast 

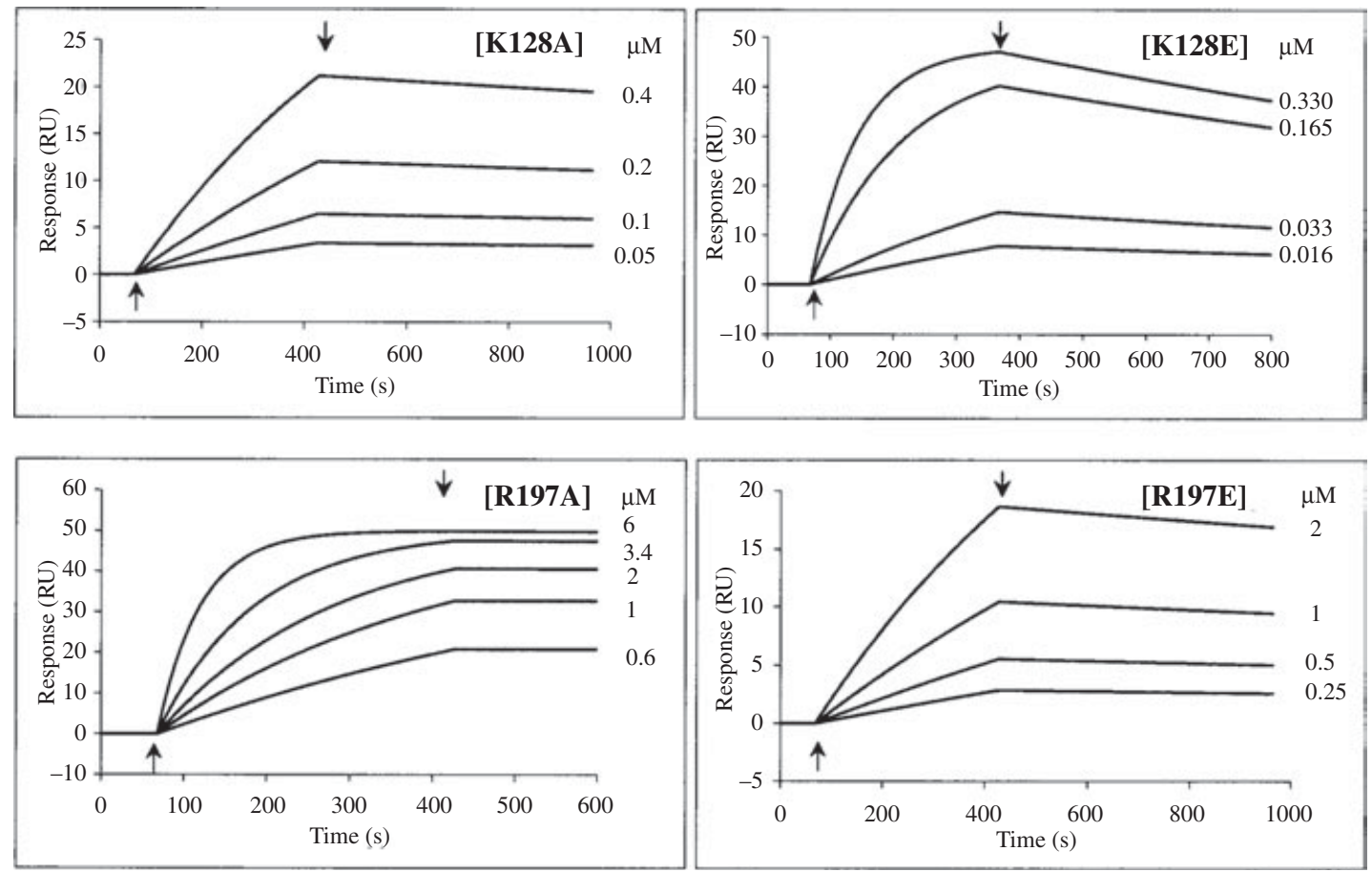

Fig. 3. Study of the interaction between GAPDH mutants and CP12 by surface plasmon resonance. Net sensorgrams (after subtracting the bulk refractive index) were obtained with immobilized CP12 using different concentrations indicated on each curve of K128A mutant GAPDH, K128E mutant GAPDH, R197A mutant GAPDH, and R197E mutant GAPDH. In all plots, the arrow on the left indicates the beginning of the association phase; the beginning of the dissociation phase is marked by the arrow on the right. The experimental data were analyzed using global fitting assuming a $1: 1$ interaction with BIAEVALUATION 3.1 .

Table 5. Dissociation constants and quantification of the destabilizing effect of the mutations on the interaction between mutant GAPDHs and CP12. The dissociation constants were measured by surface plasmon resonance with GAPDH as analyte and CP12 as ligand (immobilized protein). The free energies of the association of GAPDH and CP12 were calculated according to equations 2 and 3 in the main text.

\begin{tabular}{lccl}
\hline Analyte & $K_{\mathrm{d}}(\mathrm{nM})$ & $\Delta G_{\mathrm{b}}\left(\mathrm{kcal} \cdot \mathrm{mol}^{-1}\right)$ & $\begin{array}{l}\Delta G_{\mathrm{b}}^{\mathrm{WT}}-\Delta G_{\mathrm{b}}^{\mathrm{mut}} \\
\left(\mathrm{kcal} \cdot \mathrm{mol}^{-1}\right)\end{array}$ \\
\hline Wild-type GAPDH & 0.4 & -13.04 & \\
R197A GAPDH & 5.7 & -11.41 & -1.67 \\
R197E GAPDH & 275 & -9.09 & -3.95 \\
K128A GAPDH & 38 & -10.29 & -2.75 \\
K128E GAPDH & 14 & -10.89 & -2.14 \\
\hline
\end{tabular}

GAPDH into alanine or glutamic acid. Comparison of the kinetics of these mutants with those of the wild-type recombinant GAPDH shows that the behaviour of R197A mutant is not affected by the mutation, suggesting that the active site and the cofactor-binding site of the mutant R197A are not modified by the mutation. We thus assume that the conformation of the R197A mutant is close to that of the wild-type enzyme. In contrast, the introduction of a glutamic acid residue affects the kinetic parameters of the R197E mutant. The $K_{0.5}$ for BPGA is twice that of the wildtype recombinant GAPDH and the catalytic constant is one half with NADPH as cofactor. Residue Arg197 being located near the substrate-binding site, it is possible that the negative charge introduced with the glutamic acid could interfere with the binding of the substrate, BPGA. Replacement of the residue Lys128 results in a modification of the kinetic parameters of both the K128E and K128A mutants. They have lower catalytic rate constant and higher $K_{\mathrm{m}}$ for NADH than the wild-type GAPDH. The introduction of a negative charge does not explain the discrepancies, as the presence of an Ala residue results in the same effects, but it is possible that a slight destabilization of the region occurs, due to the absence of the positive charge on Lys128. The affinity of NADPH may be slightly altered only, because its binding depends on two hydrogen bonds between the $2^{\prime}$ phosphate group and two hydroxylated residues, Ser195 of the adjacent monomer and Ser38 (Ser188 and Thr33, respectively, on spinach GAPDH [36]).

All the different kinetic properties are probably linked to very small structural changes, as circular dichroism, fluorescence spectra and molecular modelling of all mutant GAPDHs indicate no changes of the global structure of the mutants.

To test whether the interactions of these mutants with the other partners of the GAPDH-CP12-PRK complex were impaired, we have tried to reconstitute in vitro the GAPDH-CP12 subcomplex and the GAPDH-CP12PRK complex. The K128A/E and R197A GAPDH mutants reconstitute the GAPDH-CP12 complex. Although the dissociation constants measured by surface plasmon resonance are about 10-70-fold higher than that of wild-type recombinant GAPDH and CP12, they remain low. The R197E GAPDH mutant do not reconstitute the 
subcomplex, as shown by native PAGE electrophoresis and its dissociation constant is much higher than that of the wild-type recombinant enzyme (about 600-fold). Thus, the mutation that most destabilizes the interaction with CP12 is the introduction of a negative charge at position 197. Because the introduction of an Ala residue instead of the Arg197 does not significantly impair the interaction of the R197A mutant with CP12, it seems that the mutated Arg residue is not directly involved in the interaction with the small protein. It is likely that the introduction of the negative charge destabilizes the $\mathrm{S}$ loop, thus indicating that this loop, in addition to its role in the catalytic mechanism of GAPDH could be essential for the binding of CP12. The presence of this region at the interface of GAPDH and CP12 could also explain the kinetic changes observed for the binding of the substrate when the wild-type recombinant GAPDH interacts with CP12 [28]. The differences $(\Delta \Delta \mathrm{G})$ between the binding free energy $\left(\Delta \mathrm{G}_{\mathrm{b}}\right)$ of the interaction between the wild-type GAPDH and CP12 and that of the R197E GAPDH mutant and $\mathrm{CP} 12$ is close to $-4 \mathrm{kcal} \cdot \mathrm{mol}^{-1}$. The arginine residue has the ability to form a hydrogen bond network with up to five hydrogen bonds and besides, has the ability to form a salt bridge [37] with its positively charged guanidinium group. The difference of $4 \mathrm{kcal} \cdot \mathrm{mol}^{-1}$ may correspond to the loss of the stabilizing effect of a salt bridge $[38,39]$ between an arginine residue of the S loop and CP12. This result is in good agreement with the hypothesis proposed by Sparla et al. [36], based on the kinetic and structural data obtained with a S188A mutant of $\mathrm{A}_{4}$ spinach GAPDH. This result also corroborates the idea that salt bridges in protein-protein interfaces contribute significantly to complex stabilization [26]. The possibility of a major role of salt bridges in the interaction between GAPDH and CP12 is further supported by the fact that CP12 is very rich in acidic residues, and thus has the possibility to form salt bridges with positive charges of GAPDH $[14,29]$.

Significant effects, though smaller, are also observed with the other mutations (K128A/E and R197A) for the association of the mutant GAPDHs and CP12. Most interestingly, although these mutants reconstitute the GAPDH-CP12 subcomplex, they fail to reconstitute the GAPDH-CP12-PRK complex. Two hypotheses could explain the lack of complex reconstitution. First, the mutated residues could be directly involved in the association of the GAPDH-CP12 subcomplex with PRK, but not with CP12. This would prevent the formation of half-acomplex or one unit (one tetramer of GAPDH, one dimer of PRK and one molecule of CP12) essential to the formation of the native complex by dimerization of this unit [29]. Second, we have previously shown that the association of wild-type GAPDH with CP12 resulted in a modification of the kinetic parameters of GAPDH probably through conformation changes of the enzyme upon binding of CP12 [28]. The latter were assumed to be essential for the binding of PRK by the GAPDH-CP12 subcomplex and assembly of the GAPDH-CP12-PRK complex [29]. In this case, the mutations would still enable the association of GAPDH and CP12, but would prevent or limit the conformation changes necessary to the binding of PRK. In agreement with this last hypothesis, our analysis of the kinetic properties of the GAPDH-CP12 subcomplexes obtained with the K128A/E and R197A mutants showed that the kinetic parameters were not altered upon association with CP12, unlike recombinant wild-type GAPDH [28]. They suggest that the mutations affect GAPDH conformation changes upon association with CP12, and yield a GAPDHCP12 subcomplex with considerable lower affinity for PRK, but they do not completely rule out the possibility of a direct involvement of residues K128 and R197 in the formation of the complex.

To conclude, the characterization of four GAPDH mutants (K128A/E and R197A/E) shows that the positive charges of these residues are important for the association of GAPDH and CP12, in particular, R197E mutant, and essential for the assembly of the GAPDH-CP12-PRK complex. Our results also seem to point out that the $\mathrm{S}$ loop, known to be involved in the cofactor-binding site, may also be essential for the interaction between GAPDH and CP12. Previous attempts to reconstitute the topology of the complex by cryo-electron microscopy [40] could not be achieved, partly because of the lack of information regarding the solvent-exposed regions or the interfaces between the different partners of this complex. These mutageneses are a first step toward the understanding of protein-protein interactions in the GAPDH-CP12-PRK complex and the nature of the physico-chemical forces involved in the assembly process of this higher order structure.

\section{Acknowledgements}

The authors thank Dr Owen Parkes for editing and Dr Luisana Avilan for help in preparing and for critical reading of the manuscript.

\section{References}

1. Gontero, B., Cardenas, M.L. \& Ricard, J. (1988) A functional fiveenzyme complex of chloroplasts involved in the Calvin cycle. Eur. J. Biochem. 173, 437-443.

2. Müller, B. (1972) A labile $\mathrm{CO}_{2}$-fixing enzyme complex in spinach chloroplasts. Z. Naturforsch. 27b, 925-932.

3. Nicholson, S., Easterby, J.S. \& Powls, R. (1987) Properties of a multimeric protein complex from chloroplasts possessing potential activities of NADPH-dependent glyceraldehyde-3-phosphate dehydrogenase and phosphoribulokinase. Eur. J. Biochem. 162, 423-431.

4. Anderson, L.E., Goldhaber-Gordon, I.M., Li, D., Tang, X.Y., Xiang, M. \& Prakash, N. (1995) Enzyme-enzyme interaction in the chloroplast: glyceraldehyde-3-phosphate dehydrogenase, triose phosphate isomerase and aldolase. Planta. 196, 245-255.

5. Avilan, L., Gontero, B., Lebreton, S. \& Ricard, J. (1997) Memory and imprinting effects in multienzyme complexes - I. Isolation, dissociation, and reassociation of a phosphoribulokinase-glyceraldehyde-3-phosphate dehydrogenase complex from Chlamydomonas reinhardtii chloroplasts. Eur. J. Biochem. 246, 78-84.

6. Nooren, I.M.A. \& Thornton, J.M. (2003) Diversity of proteinprotein interactions. EMBO J. 22, 3486-3492.

7. Smith, G.R. \& Sternberg, M.J. (2002) Prediction of protein-protein interactions by docking methods. Curr. Opin. Struct. Biol. 12, 28-35.

8. Gontero, B., Lebreton, S. \& Graciet, E. (2001) Protein-protein interactions in plant metabolism. In Annual Plant Review (McManus, M.T., Laing, W.A. \& Allan, A.C., eds), pp. 120-150. Sheffield Academic Press, Sheffield, UK. 
9. Romanova, A.K. \& Pavlovets, V.V. (1997) Supramolecular complexes of the enzymes participating in photosynthetic carbon dioxide assimilation. Russian J. Plant Physiol. 44, 230-238.

10. Jones, S. \& Thornton, J.M. (1996) Principles of protein-protein interactions. Proc. Natl Acad. Sci. USA 93, 13-20.

11. Glaser, F., Steinberg, D.M., Vakser, I.A. \& Ben-Tal, N. (2001) Residue frequencies and pairing preferences at protein-protein interfaces. Proteins Struct. Func. Gen. 43, 89-102.

12. Lo Conte, L., Chothia, C. \& Janin, J. (1999) The atomic structure of protein-protein recognition sites. J. Mol. Biol. 285, 2177-2198.

13. Gallet, X., Charloteaux, B., Thomas, A. \& Brasseur, R. (2000) A fast method to predict protein interaction sites from sequences. J. Mol. Biol. 302, 917-926.

14. Wedel, N., Soll, J. \& Paap, B.K. (1997) CP12 provides a new mode of light regulation of Calvin cycle activity in higher plants. Proc. Natl Acad. Sci. USA 94, 10479-10484.

15. Wedel, N. \& Soll, J. (1998) Evolutionary conserved light regulation of Calvin cycle activity by NADPH-mediated reversible phosphoribulokinase/CP12/glyceraldehyde-3- phosphate dehydrogenase complex dissociation. Proc. Natl Acad. Sci. USA 95, 9699-9704.

16. Lebreton, S., Gontero, B., Avilan, L. \& Ricard, J. (1997) Information transfer in multienzyme complexes -1 . Thermodynamics of conformational constraints and memory effects in the bienzyme glyceraldehyde-3-phosphate-dehydrogenase-phosphoribulokinase complex of Chlamydomonas reinhardtii chloroplasts. Eur. J. Biochem. 250, 286-295.

17. Lebreton, S., Gontero, B., Avilan, L. \& Ricard, J. (1997) Memory and imprinting effects in multienzyme complexes - II. Kinetics of the bienzyme complex from Chlamydomonas reinhardtii and hysteretic activation of chloroplast oxidized phosphoribulokinase. Eur. J. Biochem. 246, 85-91.

18. Lebreton, S. \& Gontero, B. (1999) Memory and imprinting in multienzyme complexes: evidence for information transfer from glyceraldehyde-3-phosphate dehydrogenase to phosphoribulokinase under reduced state in Chlamydomonas reinhardtii. J. Biol. Chem. 274, 20879-20884.

19. Lebreton, S., Graciet, E. \& Gontero, B. (2003) Modulation, via protein-protein interactions, of glyceraldehyde-3- phosphate dehydrogenase activity through redox phosphoribulokinase regulation. J. Biol. Chem. 278, 12078-12084.

20. Graciet, E., Lebreton, S., Camadro, J.M. \& Gontero, B. (2002) Thermodynamic analysis of the emergence of new regulatory properties in a phosphoribulokinase-glyceraldehyde 3-phosphate dehydrogenase complex. J. Biol. Chem. 277, 12697-12702.

21. Scagliarini, S., Trost, P., Pupillo, P. \& Valenti, V. (1993) Light activation and molecular-mass forms of NAD(P)-glyceraldehyde 3-phosphate dehydrogenase of spinach and maize leaves. Planta. 190, 313-319.

22. Baalmann, E., Backhausen, J.E., Kitzmann, C. \& Scheibe, R. (1994) Regulation of NADP-dependent glyceraldehyde 3-phosphate dehydrogenase activity in spinach chloroplast. Bot. Acta 107, 313-320.

23. Scheibe, R., Wedel, N., Vetter, S., Emmerlich, V. \& Sauermann, S.M. (2002) Co-existence of two regulatory NADP-glyceraldehyde 3-P dehydrogenase complexes in higher plant chloroplasts. Eur. J. Biochem. 269, 5617-5624.

24. Avilan, L., Gontero, B., Lebreton, S. \& Ricard, J. (1997) Information transfer in multienzyme complexes. 2. The role of Arg64 of Chlamydomonas reinhardtii phosphoribulokinase in the information transfer between glyceraldehyde-3-phosphate dehydrogenase and phosphoribulokinase. Eur. J. Biochem. 250, 296-302.

25. Bogan, A.A. \& Thorn, K.S. (1998) Anatomy of hot spots in protein interfaces. J. Mol. Biol. 280, 1-9.

26. Li, D., Urrutia, M., Smith-Gill, S.J. \& Mariuzza, R.A. (2003) Dissection of binding interactions in the complex between the antilysozyme antibody HyHEL-63 and its antigen. Biochemistry 42, $11-22$.

27. Li, Y., Li, H., Smith-Gill, S.J. \& Mariuzza, R.A. (2000) Threedimensional structures of the free and antigen-bound Fab from monoclonal antilysozyme antibody HyHEL-63. Biochemistry 39, 6296-6309.

28. Graciet, E., Lebreton, S., Camadro, J.M. \& Gontero, B. (2003) Characterization of native and recombinant A4 glyceraldehyde 3-phosphate dehydrogenase. Eur. J. Biochem. 270, 129-136.

29. Graciet, E., Gans, P., Wedel, N., Lebreton, S., Camadro, J.M. \& Gontero, B. (2003) The small protein CP12: a protein linker for supramolecular assembly. Biochemistry 42, 8163-8170.

30. Baalmann, E., Backhausen, J.E., Rak, C., Vetter, S. \& Scheibe, R. (1995) Reductive modification and nonreductive activation of purified spinach chloroplast NADP-dependent glyceraldehyde3-phosphate dehydrogenase. Arch. Biochem. Biophys. 324, 201208

31. Bradford, M.M. (1976) A rapid and sensitive method for the quantitation of microgram quantities of protein utilizing the principle of protein-dye binding. Anal. Biochem. 72, 248-254.

32. Sali, A. \& Blundell, T.L. (1993) Comparative protein modelling by satisfaction of spatial restraints. J. Mol. Biol. 234, 779-815.

33. Case, D.A., Pearlman, D.A., Caldwell, J.W., Cheatham, T.E., Ross, W.S., Simmerling, C.L., Darden, T.A., Merz, K.M., Stanton, R.V., Cheng, A.L., Vincent, J.J., Crowley, M., Tsui, V., Radmer, R.J., Duan, Y., Pitera, J., Massova, I., Seibel, G.L., Singh, U.C., Weiner, P.K. \& Koll-man, P.A. (1999) AMBER 6. University of California, San Francisco, CA.

34. Sambrook, J., Fritsch, E.F. \& Maniatis, T. (1989) Molecular Cloning: A Laboratory Manual. 2nd edn. Cold Spring Harbor Laboratory, Cold Spring Harbor, NY, USA.

35. Fermani, S., Ripamonti, A., Sabatino, P., Zanotti, G., Scagliarini, S., Sparla, F., Trost, P. \& Pupillo, P. (2001) Crystal structure of the non-regulatory A (4) isoform of spinach chloroplast glyceraldehyde-3-phosphate dehydrogenase complexed with NADP. J. Mol. Biol. 314, 527-542.

36. Sparla, F., Fermani, S., Falini, G., Zaffagnini, M., Ripamonti, A., Sabatino, P., Pupillo, P. \& Trost, P. (2004) Coenzyme site-directed mutants of photosynthetic A (4)-GAPDH show selectively reduced NADPH-dependent catalysis, similar to regulatory AB-GAPDH inhibited by oxidized thioredoxin. J. Mol. Biol. 340, 1025-1037.

37. Hendsch, Z.S. \& Tidor, B. (1994) Do salt bridges stabilize proteins? A continuum electrostatic analysis. Protein Sci. 3, 211-226.

38. Fersht, A.R. (1972) Conformational equilibria in $\alpha$ - and $\delta$-chymotrypsin: the energetics and importance of the salt bridge. J. Mol. Biol. 64, 497-509.

39. Anderson, D.E., Becktel, W.J. \& Dahlquist, F.W. (1990) pH-induced denaturation of proteins: a single salt bridge contributes 3-5 $\mathrm{kcal} / \mathrm{mol}$ to the free energy of folding of T4 lysozyme. Biochemistry 29, 2403-2408.

40. Mouche, F., Gontero, B., Callebaut, I., Mornon, J.P. \& Boisset, N. (2002) Striking conformational change suspected within the phosphoribulokinase dimer induced by interaction with GAPDH. J. Biol. Chem. 277, 6743-6749. 